\title{
“TAU-P MAPPING" BASED FILTERING TECHNIQUES FOR LINEAR NOISE ATTENUATION IN REFLECTION DATA
}

\author{
G.P.Deidda, R.Balia, Dipartimento di Ingegneria del Territorio, University of Cagliari, Italy; \\ L.Sambuelli, Dipartimento di Georisorse e Territorio, Politecnico di Torino, Italy.
}

One of the most relevant problems in reflection data processing is related to the suppression of localised coherent noises. In shallow reflection surveys typical examples among these noises are: a) the air-coupled wave, due to the extensive use of low-cost surface energy sources such as dropping weights, hammers, rifles and so on, which produce, more or less extensively, a wide spectrum, high amplitude air wave; b) other linear noises, such as the direct and refracted waves which, in addition to the fact that their frequency band overlaps significantly that of the signal, are very close to the reflections themselves and/or are spatially undersampled.

As a first consequence, particularly when low dynamic range acquisition systems such as relatively old engineering seismographs are employed, the strong amplitudes of the noise may irreparably suppress the weak amplitudes of the signal. Moreover, even when a high dynamic range apparatus is available, the undesired signal may entirely overlap the reflections both in the $\mathrm{x}-\mathrm{t}$ and $\mathrm{f}-\mathrm{k}$ planes. The air wave, in particular, due to its low velocity of propagation, is often spatially undersampled so that it shows a high order spatial aliasing in $\mathrm{f}-\mathrm{k}$ spectrum. This is, of course, a serious obstacle in an efficient $\mathrm{f}-\mathrm{k}$ filtering designing and performing. All this generally implies difficult data processing and, very often, the substantial failure of the survey, which obviously results in economic damage. In all cases, spatial aliasing of various noises, direct wave and shallow refractions can lead to erroneous interpretations (Steeples and al., 1986); the importance of removing, or at least attenuating, the air wave and the direct and refracted waves is therefore easily understandable.

In this short note a new processing procedure for attenuating linear coherent noises, even spatially aliased, which cross or are very close to the reflections, is described and proposed. It still utilises the $\tau$ - $p$ transform with the only objective of extracting linear noise from the seismic record. Reflections don't pass through slant stack operator, so as to keep their spectral 
content unchanged. Noise is removed by simply subtracting the estimated coherent linear event from the field record.

The filtering technique is based on two basic hypotheses: a) the superposition principle is valid, that is, a seismic record results from superposition of several events (direct, refracted, reflected, coherent and random noises); b) a linear event in the $x$ - $t$ domain maps in a point in the $\tau$ - $p$ domain and viceversa. The first hypothesis always proves correct while the other is true only when the data are not sampled and when they have infinite spatial aperture (Yilmaz et al., 1994). In fact, the desired transform point is usually smeared with linear artefacts because of the relatively small aperture of the input data and their sampled nature. The point smearing can be significantly decreased in several ways; for example, one can apply a p-direction deconvolution (Zhou et al., 1994). In the present paper this goal is achieved with a procedure that is synthetically shown in the block diagram as well as described below.

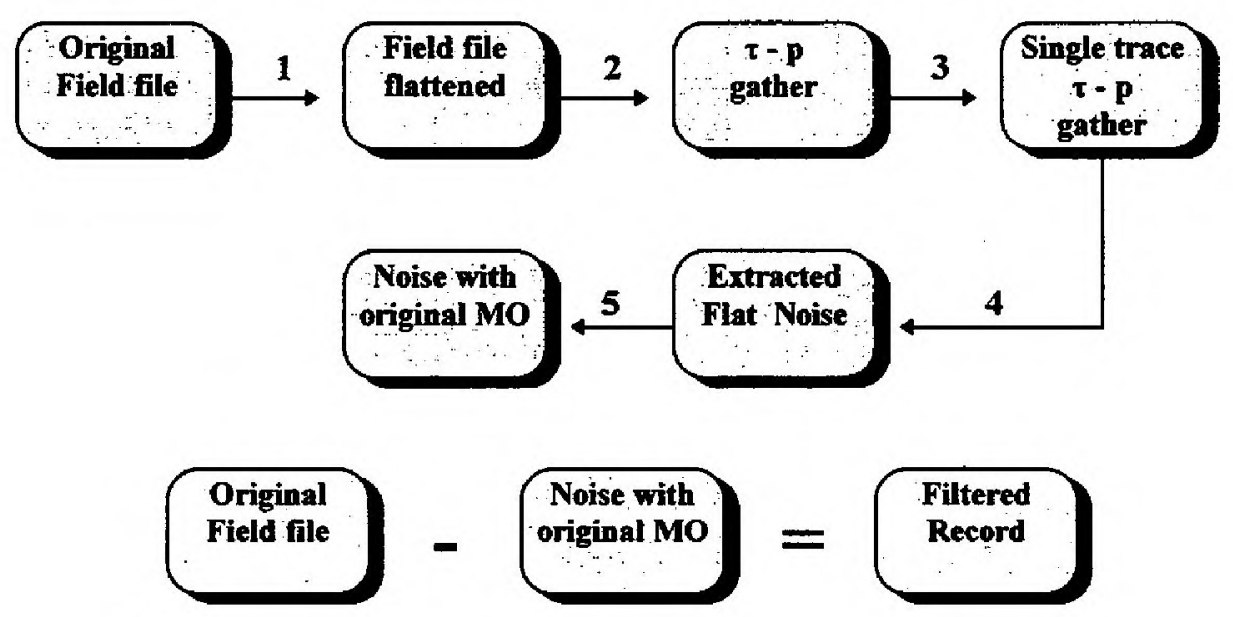

1. After determining the velocity and the zero-offset time $\left(T_{x}=0\right)$ of the linear event to be removed, this is flattened by application of an LMO (linear moveout) with a velocity equal to the estimated one, so that the event has an infinite apparent velocity.

2. Using slant stack the record with new moveout characteristics is transformed from $x-t$ to $\tau-p$ domain. The noise is not spatially aliased, since it has infinite apparent velocity and so it is correctly mapped in $\tau$-p. Artefacts due to spatial truncation (windowing effects) and small aperture of the input data are likely to be present.

In order to avoid aliasing in the p-direction the slant stack is carried out with a very high minimum velocity (for e.g. 100,000 unit distance/s) and a high number of ray-parameter.

Given these conditions, the linear events in $x-t$ have an image in $\tau$-p very close to a point (a band limited signal starting at $\tau=T_{x=0}$ focused on trace $p=0$ and those nearest to it). 
3. A surgical muting is applied so that samples are zeroed for all traces with $p \neq 0$, whereas for trace with $\mathrm{p}=0$ only a window beginning in $\tau=\mathrm{T}_{\mathrm{x}=0}$ and having length equal to noise duration remains.

4. The inverse $\tau$ - p transform is then executed, whose result is the estimated noise, still flattened.

5. Using an inverse LMO the original moveout characteristics are then re-established.

At this point of the procedure, comparing for difference the original field record to the record with estimated noise the filtered record is obtained.

If a certain amount of noise is still present, the whole procedure can be repeated over and over until filtering is adequate. Before performing step 2, any trace to trace time and amplitude variations are removed by correcting for static shifts and applying the gain factors. The latter ones are saved and recovery scaling is subsequently applied to the noise estimate, thus preserving true amplitude. The effectiveness of this procedure have been tested on a set of both synthetic and real data. The procedure is particularly suitable for utilisation on high resolution shallow reflection seismic data, since it doesn't alter the spectral content of the signal. Finally, it allows larger geophone spacing, because it can successfully filter out spatial aliased coherent noises, so that acquisition and processing time can be saved.

\section{REFERENCES}

Kelamis P.G. and Mitchell A.R., 1989. Slant-stack processing. First Break, 2.

Steeples W. and Miller R.D., 1986. Some Shallow Seismic Reflection Pitfalls. 56th Annual SEG Meeting, Houston, Expanded Abstracts: 101-104.

Turner G., 1990. Aliasing in the $\tau$ - p transform and the removal of spatially aliased coherent noise. Geophysics, 55: 1496-1503.

Yilmaz O. and Taner T., 1994. Discrete plane-wave decomposition by least-mean-squareerror method. Geophysics, 59: 973-982.

Zhou B. and Greenhalgh S.A., 1994. Linear and parabolic $\tau$ - p transforms revisited. Geophysics, 59: 1133-1149. 
